\title{
Crocin exerts anti-inflammatory and anti-catabolic effects on rat intervertebral discs by suppressing the activation of JNK
}

\author{
KANG LI* ${ }^{*}$ YAN LI* ${ }^{*}$ ZHENJIANG MA and JIE ZHAO \\ Shanghai Key Laboratory of Orthopaedic Implants, Department of Orthopaedic Surgery, \\ Shanghai Ninth People's Hospital, Shanghai Jiao Tong University School of Medicine, Shanghai 200011, P.R. China
}

Received March 26, 2015; Accepted September 17, 2015

DOI: $10.3892 /$ ijmm.2015.2359

\begin{abstract}
As intervertebral disc (IVD) degeneration has been proven to contribute to low back pain (LBP), drug treatment aiming at attenuating IVD degeneration may prove to be benefiical. Crocin, a bioactive component of saffron, has been found to exert anti-inflammatory effects on cartilage. In the present study, the anti-inflammatory and anti-catabolic effects of crocin on rat IVDs were analyzed in vitro and ex vivo. Nucleus pulposus (NP) cells were isolated from the lumbar IVDs of Sprague-Dawley rats. The NP cells were first treated with various concentrations of crocin, and then stimulated with lipopolysaccharide (LPS) to induce inflammation. Subsequently, RT-qPCR and enzymelinked immunosorbent assay were carried out to measure the expression levels of catabolic enzymes, pro-inflammatory factors and the components of the extracellular matrix (ECM). In addition, western blot analysis was also used to investigate the related signaling pathways. The whole spinal motion segment (vertebra-IVD-vertebra section) of the rats was isolated and cultured in the presence or absence of LPS and crocin for 7 days. The ex vivo effects of crocin on the ECM of the IVD structures were determined by histological and biochemical analysis. In vitro, crocin significantly inhibited the LPS-induced overexpression of catabolic enzymes [matrix metalloproteinase (MMP)-1, MMP-3, MMP-13, a disintegrin-like and metalloprotease (reprolysin type) with thrombospondin type 1 motif (ADAMTS)-4 and ADAMTS-5], pro-inflammatory factors [interleukin (IL)-1 $\beta$, tumor necrosis factor (TNF)- $\alpha$, IL-6 and inducible nitric oxide synthase (iNOS)] and Toll-like receptor (TLR)-2 in a concentration-dependent manner. Notably, crocin partly prevented the downregulation of aggrecan and type II collagen (collagen-II). Moreover, crocin suppressed
\end{abstract}

Correspondence to: Professor Jie Zhao, Shanghai Key Laboratory of Orthopaedic Implants, Department of Orthopaedic Surgery, Shanghai Ninth People's Hospital, Shanghai Jiao Tong University School of Medicine, 639 Zhizaoju Road, Shanghai 200011, P.R. China E-mail: spinezhaojie@163.com

*Contributed equally

Key words: crocin, nucleus pulposus, inflammation, lipopolysaccharide, c-Jun $\mathrm{N}$-terminal kinase, organ culture the LPS-induced activation of the mitogen-activated protein kinase (MAPK) pathway by inhibiting the phosphorylation of c-Jun N-terminal kinase (JNK). Ex vivo experiments demonstrated that crocin protected the rat IVDs from the LPS-induced depletion of the ECM components, including proteoglycan and collagen-II. In conclusion, crocin effectively suppressed the degeneration-related inflammation and catabolism in rat IVDs in vitro and ex vivo, suggesting that crocin has potential for use as a therapuetic strategy in the treatment of LBP.

\section{Introduction}

Low back pain (LBP), one of the most common musculoskeletal complaints, has become a serious health and socioeconomic problem affecting modern society $(1,2)$. Among numerous pain generators in the lumbar spine, intervertebral disc (IVD) degeneration is considered to be the most important contributor to LBP $(3,4)$. IVD degeneration results from the excessive degradation and impaired synthesis of the extracellular matrix (ECM) secreted by nucleus pulposus (NP) cells. This imbalance between catabolic and anabolic activity leads to changes in the biological and mechanical characteristics of IVDs (5-11).

A number of previous studies have proven that IVD degeneration is linked to various pro-inflammatory cytokines and metabolites, including interleukin (IL)-1 $\beta$, IL-6, tumor necrosis factor- $\alpha(\mathrm{TNF}-\alpha)$, lipopolysaccharide (LPS) and reactive oxygen species (ROS) (12-17). LPS, an endotoxin which exists in the outer membrane of Gram-negative bacteria, has been used to induce inflammatory responses in several pre-clinical models of inflammation $(18,19)$. There is evidence to indicate that LPS is a potential inducer of multiple pro-inflammatory cytokines (IL-1 $\beta$, IL-6 and TNF- $\alpha$ ) through binding to Tolllike receptor (TLR)-2 and TLR-4 (18,20-24). In cartilaginous tissue, the mitogen-activated protein kinases (MAPK) pathway, with p38, extracellular signal-regulated kinase (ERK) and c-Jun N-terminal kinase (JNK) as major members, is an important downstream inflammatory pathway. The results from previous studies on cartilage degeneration have indicated that the MAPK pathway plays a pivotal role in the inflammatory process $(25,26)$. Current treatment strategies for LBP are limited to conservative treatment (e.g., physical therapy and oral analgesics) and surgical intervention (27-29); anti-inflammatory and regenerative drug treatment has not been applied to clinical practice. Although few studies have focused on the application 
of plant-derived compounds to attenuate IVD degeneration, some anti-inflammatory candidates may provide us with novel therapeutic strategeis for the treatment of LBP.

Crocus sativus L. (or saffron), which belongs to the Iridaceae family, is often used as a culinary spice or an anodyne or tranquilizer in traditional Chinese medicine. Crocin is the main constituent which is responsible for the multiple bioactivities of saffron. Previous studies have demonstrated the anti-inflammatory effects of crocin which are mediated through the inhibition of nitric oxide (NO) synthesis in LPS-induced inflammation (30-32). Crocin has recently been reported to exert an anti-arthritic effect on cartilaginous tissues, suggesting that crocin protects cartilage from IL-1 $\beta$-induced inflammation by suppressing the expression of matrix metalloproteinases (MMPs) (33).

Therefore, in this study, we aimed to further explore the reported anti-inflammatory effects of crocin by investigating the role of crocin in inflammation induced by LPS in the NP. In the present study, inflammation-related proteolytic enzymes and pro-inflammatory factors involved in IVD degeneration were explored as inflammatory markers. A disc organ culture system, serving as an ex vivo model of IVD degeneration, was used to conduct histological and biochemical assays. Furthermore, we investigated the potential mechanisms responsible for the anti-inflammatory effects of crocin by analyzing the relevant signaling pathways.

\section{Materials and methods}

Reagents and antibodies. The chemical reagents, including crocin, used in the present study were purchased from Sigma-Aldrich (St. Louis, MO, USA) unless stated otherwise. Stock solution of crocin was prepared in phosphate-buffered saline (PBS) and stored at $-20^{\circ} \mathrm{C}$. The MAPK family antibody sampler kit (Cat. no. 9926) and phospho-MAPK family antibody sampler kit (Cat. no. 9910) were obtained from Cell Signaling Technology, Inc. (Beverly, MA, USA). Anti-type II collagen (collagen-II) antibody (ab34712) was purchased from Abcam (Cambridge, MA, USA).

$N P$ cell isolation and culture. The present study was conducted in strict accordance with the recommendations in the Guide for the Care and Use of Laboratory Animals of the National Institutes of Health. The study protocol was approved by the Animal Care and Experiment Committee of Shanghai Jiao Tong University School of Medicine, Shanghai, China. A total of 30 Sprague-Dawley rats ( 3 months old) were provided by the Experimental Animal Center of Shanghai Ninth People's Hospital (Shanghai, China). Lumbar IVDs were harvested from the rats immediately following sacrifice (by intraperitoneal injection of excessive chloral hydrate). Under a magnifier, the NP tissues were carefully removed without adhesion of the annulus fibrosus and endplate from the IVD. To ensure the purity of the NP cells, the NP tissues were rinsed with DMEM/F-12 medium many times until all impurities were removed. The NP tissues were then dissected into $1-\mathrm{mm}^{3}$ sections for sufficient digestion. The NP sections were digested in $0.25 \%$ trypsin (Sigma-Aldrich) for $15 \mathrm{~min}$ and $0.025 \%$ type II collagenase (Sigma-Aldrich) for $4 \mathrm{~h}$ at $37^{\circ} \mathrm{C}$. Tissues debris was removed from the digestion product using a $70-\mu \mathrm{M}$ filter. The NP cells were cultured in DMEM/F-12 medium containing 10\% fetal bovine serum (FBS) and $1 \%$ penicillin/streptomycin at $37^{\circ} \mathrm{C}$, in a $5 \% \mathrm{CO}_{2}$ humidified incubator. When the primary cells reached a confluence of $80-90 \%$, they were harvested, replated and allowed to proliferate. The second-generation cells were used throughout the experiments.

Cytotoxicity assay. Cell viability was measured with a Cell Counting Kit-8 (CCK-8; Dojindo Laboratories, Kumamoto, Japan) according to the instructions provided by the manufacturer. Briefly, the rat NP cells were seeded in 96-well plates at a density of $5 \times 10^{3}$ cells/well, and then incubated with various concentrations of crocin $(10,50,100$ and $200 \mu \mathrm{M})$ for $24 \mathrm{~h}$. CCK-8 solution $(10 \mu \mathrm{l})$ was added to each well and the plates were incubated for $2 \mathrm{~h}$. The absorbance of each well was measured at $450 \mathrm{~nm}$ using a microplate reader (Omega Bio-Tek, Inc., Norcross, GA, USA) with the culture medium as a blank. Cell viability was calculated using the following formula: cell viability $(\%)=$ [absorbance (with crocin) - absorbance (blank)]/ [absorbance (without crocin) - absorbance (blank)] x100.

Cell treatment. The NP cells were seeded in 6-well plates at a density of $1 \times 10^{5}$ cells/well. After reaching a confluence of $80-90 \%$, the cells were treated with or without various concentrations of crocin $(10,50$ and $100 \mu \mathrm{M})$ for $2 \mathrm{~h}$, followed by stimulation with LPS (Sigma-Aldrich; $10 \mu \mathrm{g} / \mathrm{ml}$ ) for $24 \mathrm{~h}$. The cells were harvested for use in an expression assay of IVD degeneration-related genes. Supernatants were collected for the evaluation of MMP-3 and MMP-13 expression by enzyme-linked immunosorbent assay (ELISA). For western blot analysis, the cells were pre-treated with crocin for $4 \mathrm{~h}$, and total proteins were extracted $30 \mathrm{~min}$ following stimulation with LPS.

Isolation of RNA and reverse transcription-quantitative polymerase chain reaction ( $R T-q P C R)$. Total RNA was isolated using TRIzol reagent (Invitrogen, Carlsbad, CA, USA) following the manufacturer's instructions. Reverse transcription was carried out with $1 \mu \mathrm{g}$ of total RNA using a reverse transcriptase kit (Takara Bio, Inc., Otsu, Japan) for first-strand complementary DNA (cDNA) synthesis. Quantitative (real-time) PCR (qPCR) was conducted with a SYBR Premix Ex Taq kit (Takara Bio, Inc.) and an ABI 7500 Sequencing Detection System (Applied Biosystems/Life Technologies, Foster City, CA, USA) according to the manufacturer's instructions. Relative gene expression was measured using the $\Delta \Delta \mathrm{Ct}$ method with $\beta$-actin as an internal control. The primer sequences are presented in Table I.

ELISA. As described above, following pre-treatment with various concentrations of crocin $(0,10,50$ and $100 \mu \mathrm{M})$ for $2 \mathrm{~h}$, the NP cells were stimulated with LPS $(10 \mu \mathrm{g} / \mathrm{ml})$ for $24 \mathrm{~h}$. The supernatants were then collected and centrifuged to remove cell fragments. The levels of MMP-3 and MMP-13 in the supernatant were measured using an ELISA kit (R\&D Systems, Inc., Minneapolis, MN, USA) and a microplate reader (Omega Bio-Tek, Inc.) according to the manufacturer's instructions.

Western blot analysis. Total protein was extracted using radioimmunoprecipitation assay (RIPA) lysis buffer containing a mixture of protease inhibitor, and the protein amount was determined by the Bradford method. The denatured protein was separated by sodium dodecyl sulfate-polyacrylamide 
Table I. Sequences of primers used for RT-qPCR.

Gene $\quad$ Primer sequences (5'-3')

MMP-1

F: AGCTCATACAGTTTCCCCGT R: GCCTCAGCTTTTCAGCCATC

MMP-3

F: TTTGGCCGTCTCTTCCATCC R: GCATCGATCTTCTGGACGGT

MMP-13

F: ACCATCCTGTGACTCTTGCG R: TTCACCCACATCAGGCACTC

ADAMTS-4

F: ACCGATTACCAGCCTTTGGG R: CCGACTCCGGATCTCCATTG

ADAMTS-5

F: CCGAACGAGTTTACGGGGAT R: TGTGCGTCGCCTAGAACTAC

IL-1 $\beta$ F: GCACAGTTCCCCAACTGGTA R: GGAGACTGCCCATTCTCGAC

IL-6 F: CATTCTGTCTCGAGCCCACC R: AGTCTCCTCTCCGGACTTGT

TNF- $\alpha$ F: TCGTAGCAAACCACCAAGCA R: TCGTAGCAAACCACCAAGCA iNOS F: ACACAGTGTCGCTGGTTTGA R: AGAAACTTCCAGGGGCAAGC

TLR-2

F: TGGAGGTCTCCAGGTCAAATC R: TGTTTGCTGTGAGTCCCGAG

Aggrecan F: CAGATGGCACCCTCCGATAC R: GACACACCTCGGAAGCAGAA

Collagen-II F: GGCCAGGATGCCCGAAAATTA R: ACCCCTCTCTCCCTTGTCAC

$\beta$-actin $\quad \mathrm{F}$ : AACCTTCTTGCAGCTCCTCCG R: CCATACCCACCATCACACCCT

F, forward; R, reverse.

gel electrophoresis (SDS-PAGE), transferred onto PVDF membranes (Millipore Corp., Billerica, MA, USA) and probed with the primary antibodies against p38, p-p38, ERK, p-ERK, JNK, p-JNK and $\beta$-actin. Following incubation with the appropriate secondary antibodies conjugated with IRDye $800 \mathrm{CW}$ (molecular weight $1162 \mathrm{Da}$ ), bands of interest were detected by exposure using an Odyssey infrared imaging system (LI-COR Biosciences, Lincoln, NE, USA). Quantitative analysis of the intensity of bands was done using Adobe Photoshop CS6 (Adobe Systems Incorporated, San Jose, CA, USA).

Organ culture. An extra 10 Sprague-Dawley rats were euthanized by an injection of a lethal dose of chloral hydrate. The entire discs were harvested, including the vertebral endplates, annulus fibrosus and NP, and then placed into a 12-well plate. The discs were cultured in DMEM/F-12 medium containing $10 \%$ FBS, $1 \%$ insulin-transferrin-selenium (Sigma-Aldrich), $50 \mu \mathrm{g} / \mathrm{ml}$ ascorbate-2-phosphate and $1 \%$ penicillin/strep- tomycin. The medium was replaced every day. Additional $\mathrm{NaCl}$ was added to the medium to raise the osmolarity to $410 \mathrm{mOsm} / \mathrm{kg}$, as previously described $(34,35)$. In different groups, the discs were cultured for 7 days with or without $10 \mu \mathrm{g} / \mathrm{ml}$ LPS and $100 \mu \mathrm{M}$ crocin. Moreover, at the end of the culture period, the discs were treated with $0.75 \mathrm{mg} / \mathrm{ml}$ nitroblue tetrazolium (NBT; Sigma-Aldrich) in fresh complete medium and incubated at $37^{\circ} \mathrm{C}$ for $24 \mathrm{~h}$.

Histological analysis. Following 7 days of culture, the discs were harvested, fixed in $4 \%$ paraformaldehyde and decalcified in EDTA for 14 days. The decalcified discs were embedded in paraffin for sectioning. Serial mid-sagittal sections of discs (5- $\mu \mathrm{m}$-thick) were obtained to prepare slides. NBT and 4,6-diamino-2-phenylindole (DAPI; Sigma-Aldrich) dual staining was used to evaluate cell viability in the organ cultures as previously described (36). NBT staining was imaged under transmitted light illumination using a microscope (LEICA DM4000 B; Leica Microsystems, Wetzlar, Germany) and DAPI staining was imaged under ultraviolet illumination $(359 \mathrm{~nm})$ using the same microscope (LEICA DM4000 B; Leica Microsystems). Viable cells were defined as cells with both DAPI and NBT staining, and cells with DAPI staining alone were deemed dead. A total of 3 sections of each disc were analyzed and 3 fields (magnification, x400) of each section were selected to calculate the average. Cell viability was calculated as follows: cell viability $(\%)=$ live cells in field (NBT stains)/total cells in field (DAPI signal) x100. Mid-sagittal sections were also stained with hematoxylin-eosin (H\&E) and Safranin O-fast green stain to assess the degeneration of IVDs. All H\&E staining and Safranin O-fast green staining slides were imaged under transmitted light illumination using a microscope (LEICA DM4000 B; Leica Microsystems). All staining images were observed and assessed by an unblinded investigator, while the evaluation of the content of the ECM was based on staining intensity in the ECM area. Collagen-II expression was detected by immunohistochemistry (IHC) using a mouse monoclonal antibody (ab34712; 1:200; Abcam) and a horseradish peroxidaseconjugated anti-mouse antibody (K5007; 1:50; Dako, Glostrup, Denmark), followed by color development with diaminobenzidine tetrahydrochloride (DAB; Dako). The results of collagen-II staining were quantified and the integrated optical density (IOD) was determined using Image Pro-Plus (IPP) version 6.0 software (Media Cybernetics, Inc., Bethesda, MD, USA).

Proteoglycan content assay. Following 7 days of culture, NP tissue was isolated from each disc and then digested with papain at $37^{\circ} \mathrm{C}$ for $48 \mathrm{~h}$ (37). The proteoglycan content of the papain digests was determined by the dimethylmethylene blue (DMMB) assay as previously described (38). The DMMB assay was standardized by the dsDNA content of the NP tissue, which was determined by an assay of the total dsDNA content using the Quant-iT ${ }^{\mathrm{TM}}$ PicoGreen ${ }^{\circledR}$ dsDNA Assay kit (Invitrogen).

Statistical analysis. All experiments were repeated at least 3 times. The results are expressed as the means \pm standard deviation (SD). Statistical analysis was performed with a one-way analysis of variance (ANOVA) for multiple comparisons using SPSS 19.0 software (IBM, Corp., Armonk, NY, USA). A value of $\mathrm{P}<0.05$ was considered to indicate a statistically significant difference. 


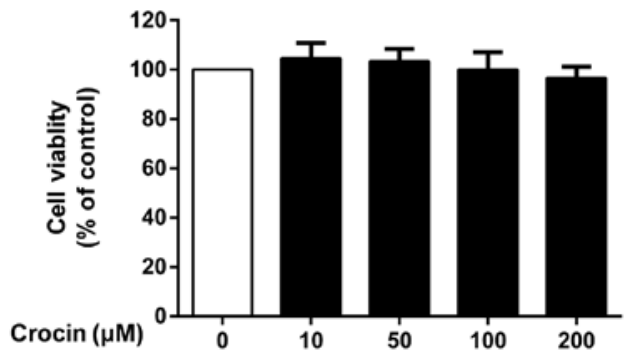

Figure 1. Effects of crocin on nucleus pulposus (NP) cell viability were evaluated by CCK- 8 assay. No significant cytotoxicity was observed following treatment with increasing concentrations $(10-200 \mu \mathrm{M})$ of crocin for $24 \mathrm{~h}$. Results are expressed as the means $\pm \mathrm{SD}(\mathrm{P}>0.05)$.

\section{Results}

Potential cytotoxicity of crocin. To determine the potential cytotoxicity of crocin, the NP cells were incubated with various concentrations $(10,50,100$ and $200 \mu \mathrm{M})$ of crocin for $24 \mathrm{~h}$ prior to performing a CCK-8 assay. Crocin did not exhibit any significant cytotoxic effects at concentrations of 10-200 $\mu \mathrm{M}$ (P>0.05; Fig. 1).

Crocin inhibits the LPS-induced increase in the mRNA expression of catabolic enzymes and pro-inflammatory factors in NP cells. As shown by RT-qPCR, the upregulated mRNA expression of MMPs (MMP-1, MMP-3 and MMP-13) and a disintegrin-like and metalloprotease (reprolysin type) with thrombospondin type 1 motif (ADAMTS; ADAMTS-4 and ADAMTS-5) induced by LPS was significantly inhibited by crocin in a concentration-dependent manner (Fig. 2A-E). However, the lowest concentration $(10 \mu \mathrm{M})$ of crocin used did not significantly inhibit the increase in the expression of ADAMTS-4 and ADAMTS-5 induced by LPS (Fig. 2D and E). Similarly, LPS markedly increased the mRNA expression of pro-inflammatory and oxidative stress factors [IL-1 $\beta$, TNF- $\alpha$, IL- 6 and inducible NO synthase (iNOS)]; this increase was significantly inhibited by pre-treatment with crocin in a concentration-dependent manner (Fig. 2F-I). Moreover, crocin partly reversed the LPS-induced decrease in the mRNA expression of aggrecan and collagen-II at the concentration of 50 and $100 \mu \mathrm{M}$ (Fig. 2J and K). Notably, the overexpression of TLR-2 induced by LPS was also significantly suppressed by pre-treatment with crocin (Fig. 2L).

Crocin inhibits the LPS-induced increase in the protein expression of MMP-3 and MMP-13 in NP cells. Consistent with the results obtained for the mRNA expression profiles, the results obtained by ELISA revealed that stimulation with LPS markedly increased the protein expression of MMP-3 and MMP-13 in the NP cells. Compared with LPS-stimulated group, treat-
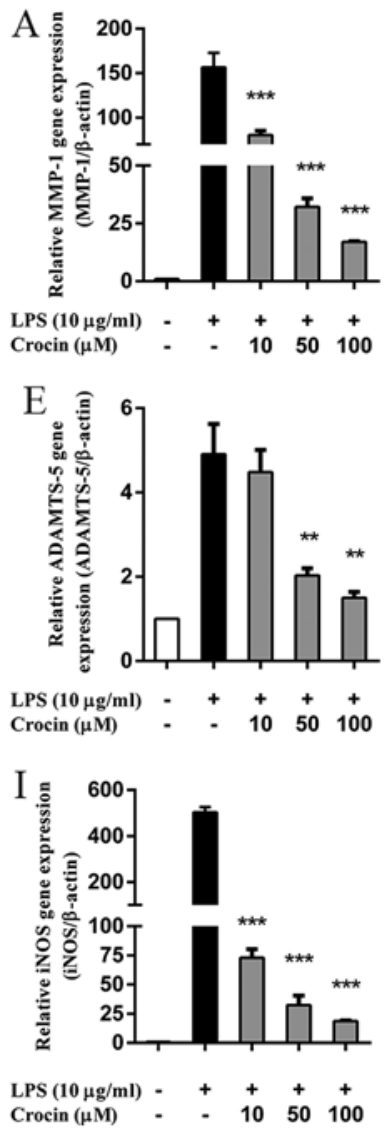
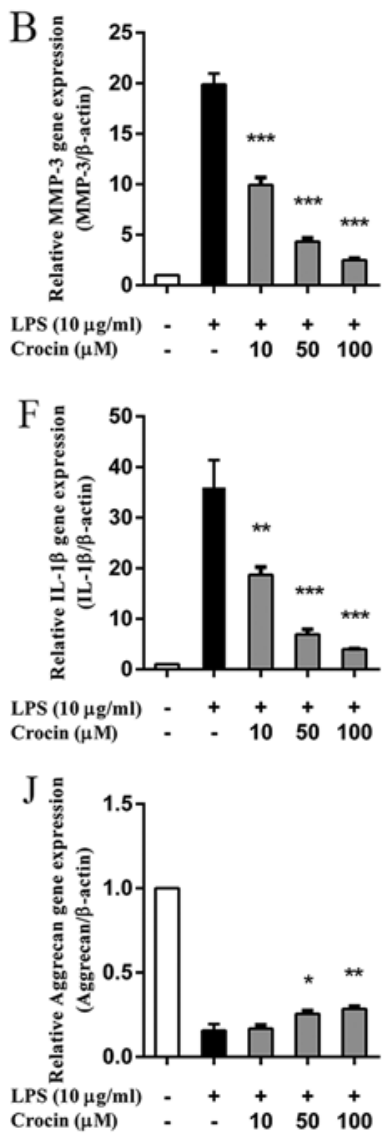
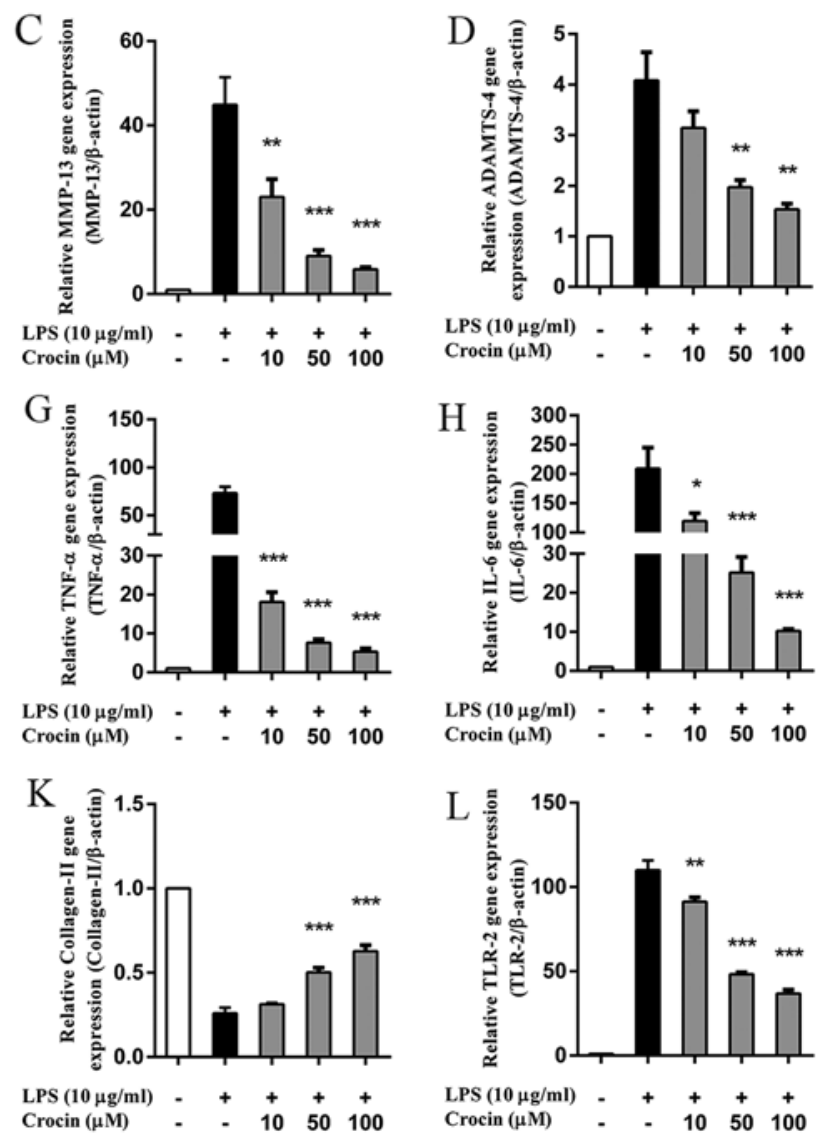

Figure 2. Effects of crocin on the lipopolysaccharide (LPS)-induced increase in the mRNA expression of intervertebral disc (IVD) degeneration-related genes. (A-L) The relative mRNA expression of (A) MMP-1, (B) MMP-3, (C) MMP-13, (D) ADAMTS-4, (E) ADAMTS-5, (F) IL-1 $\beta$, (G) TNF- $\alpha$, (H) IL-6, (I) iNOS, (J) aggrecan, (K) collagen-II and (L) TLR-2 was evaluated by RT-qPCR. Following treatment with various concentrations of crocin (10, 50 and $100 \mu \mathrm{M})$ for $2 \mathrm{~h}$, nucleus pulposus (NP) cells were stimulated with LPS $(10 \mu \mathrm{g} / \mathrm{ml})$ for $24 \mathrm{~h}$ and then harvested for us in RT-qPCR. Results are expressed as the means \pm SD. ${ }^{*} \mathrm{P}<0.05,{ }^{* *} \mathrm{P}<0.01,{ }^{* * *} \mathrm{P}<0.001$, compared to the LPS-stimulated group. 

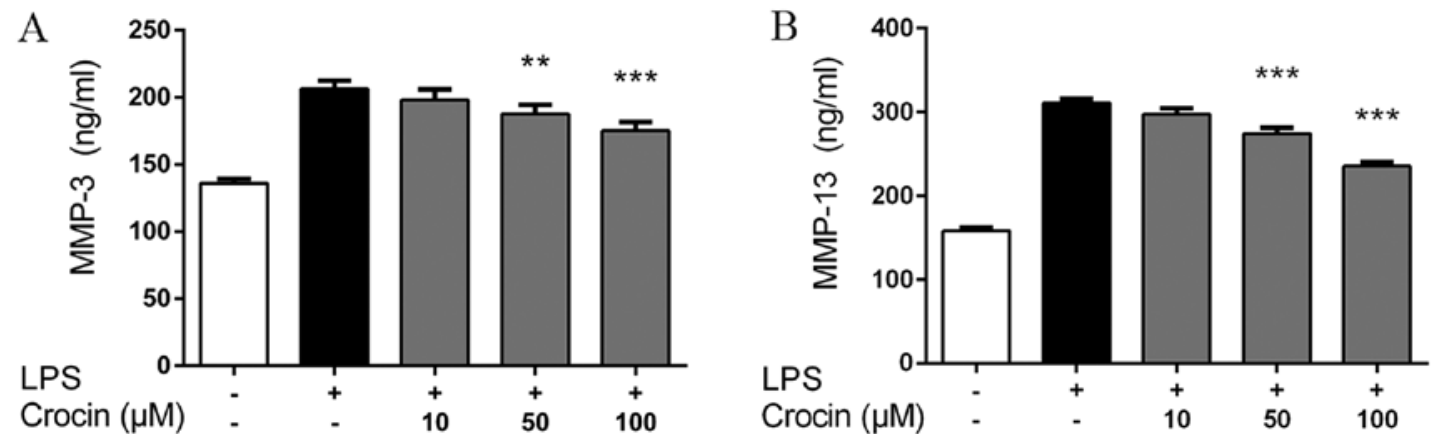

Figure 3. Effects of crocin on lipopolysaccharide (LPS)-induced protein expression of MMP-3 and MMP-13 determined by ELISA. Following treatment with various concentrations of crocin $(10,50$ and $100 \mu \mathrm{M})$ for $2 \mathrm{~h}$, nucleus pulposus (NP) cells were stimulated with LPS (10 $\mu \mathrm{g} / \mathrm{ml})$ for $24 \mathrm{~h}$ and then the supernatant was harvested for ELISA. (A) Protein levels of MMP-3 in the supernatant. (B) Protein levels of MMP-13 in the supernatant. Results are expressed as the means $\pm \mathrm{SD} .{ }^{* *} \mathrm{P}<0.01,{ }^{* * *} \mathrm{P}<0.001$, compared to the LPS-stimulated group.

A
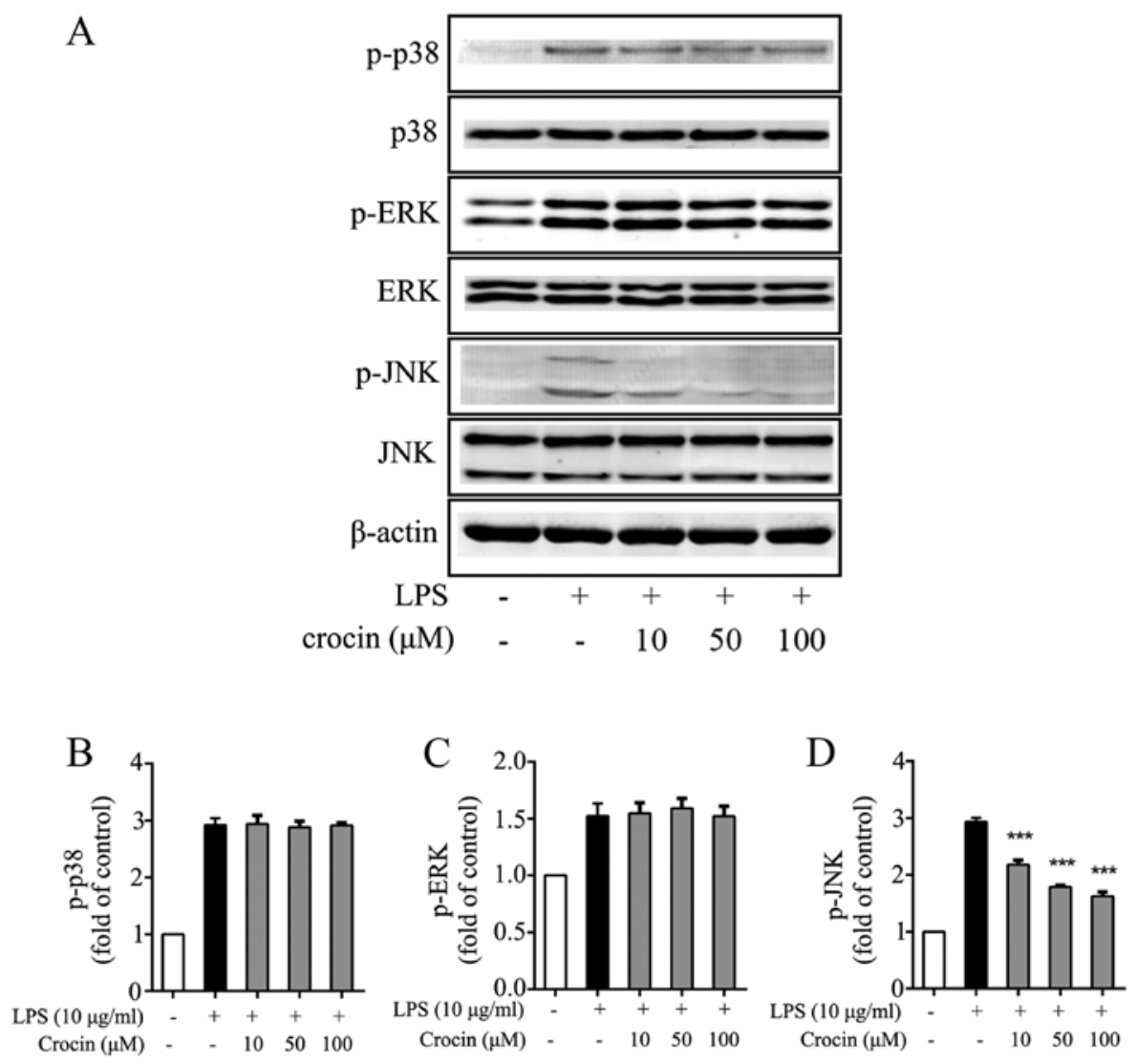

Figure 4. Effects of crocin on the phosphorylation of the MAPK protein family (p38, ERK and JNK) in nucleus pulposus (NP) cells. Following treatment with various concentrations of crocin $(10,50$ and $100 \mu \mathrm{M})$ for $4 \mathrm{~h}, \mathrm{NP}$ cells were stimulated with lipopolysaccharide (LPS) $(10 \mu \mathrm{g} / \mathrm{ml})$ for $30 \mathrm{~min}$ and then harvested for western blot analysis. (A) Western blots for p38, p-p38, ERK, p-ERK, JNK, p-JNK and $\beta$-actin. (B) Quantitative analysis of p-p38. (C) Quantitative analysis of p-ERK. (D) Quantitative analysis of p-JNK. Results are expressed as the means \pm SD. ${ }^{* * * *} \mathrm{P}<0.001$, compared to the LPS-stimulated group.

ment with crocin at 50 and $100 \mu \mathrm{M}$ significantly inhibited the LPS-induced increase in the protein expression of MMP-3 and MMP-13. No signfiicant effects were observed following pretreatment with crocin at the concentration of $10 \mu \mathrm{M}$ (Fig. 3).

Crocin inhibits the activation of JNK induced by LPS. Growing evidence supports the involvement of the MAPK signaling pathway in the inflammation and degeneration of IVDs (39-41). Hence, the ability of crocin to inhibit the activation of the MAPK signaling pathway in NP cells was investigated by western blot analysis. As expected, LPS significantly induced the phosphorylation of p38,ERK and JNK (Fig. 4). The phosphorylation of JNK was potently attenuated by treatment with crocin (Fig. 4A and D), while the levels of p-p38 and p-ERK were not obviously affected (Fig. 4A-C). The results of western blot analysis demonstrated that crocin inhibited the LPS-induced activation of the MAPK signaling pathway through JNK, and not p38 or ERK1/2.

Crocin inhibits the LPS-induced degeneration of rat IVDs in organ culture. By contrast, as shown in $\mathrm{H} \& \mathrm{E}$ staining images, 
A

A
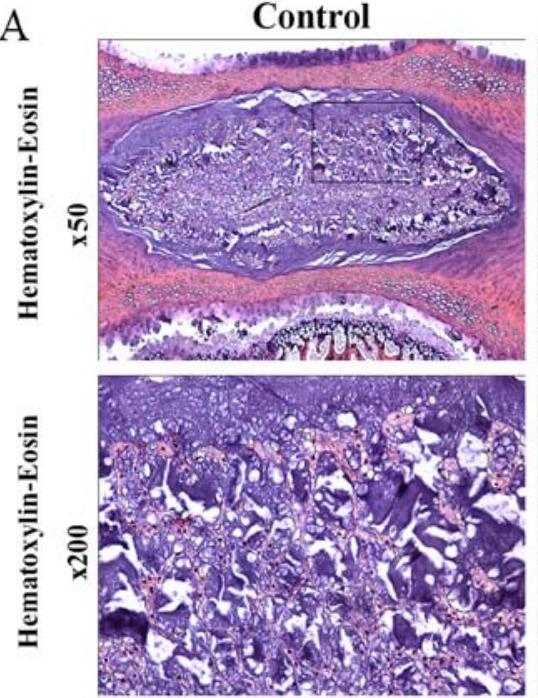

B
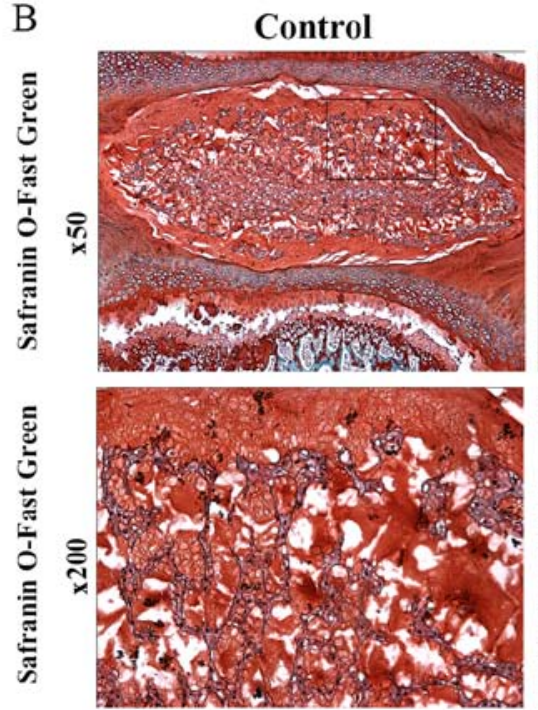

LPS
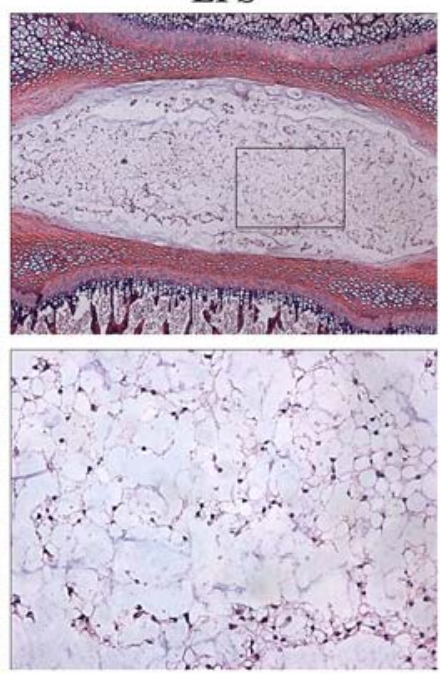

LPS
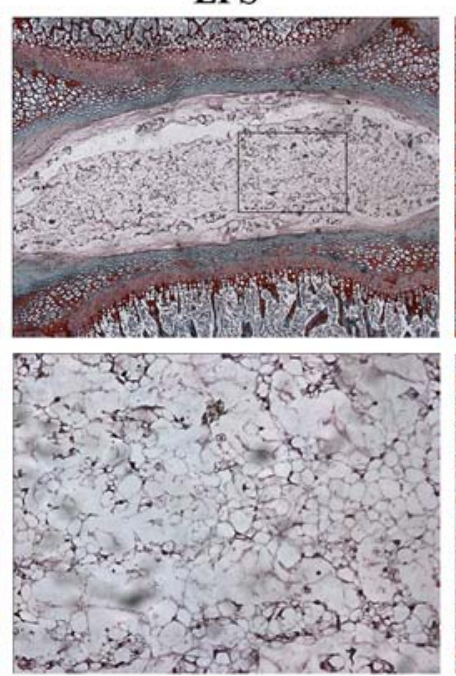

LPS+Crocin
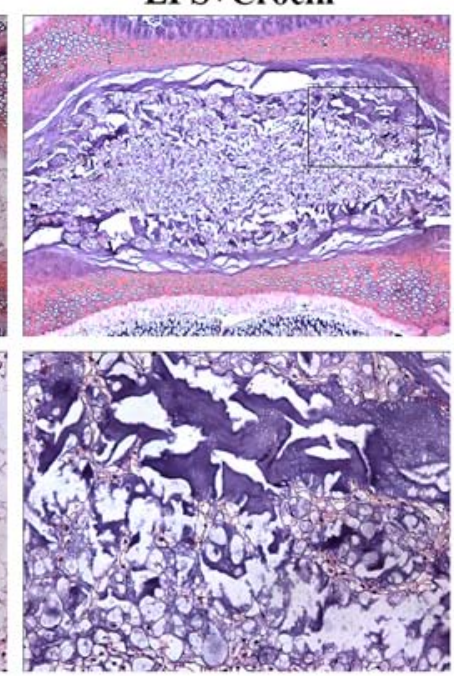

LPS+Crocin
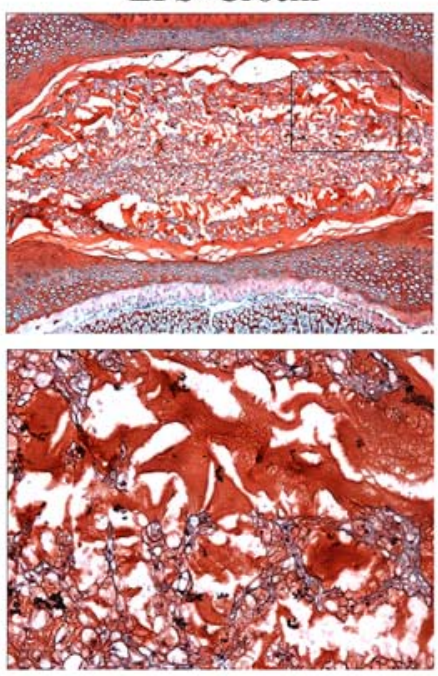

Figure 5. Histological analysis of intervertebral discs (IVDs) cultured ex vivo for the assessment of IVD degeneration. (A) Hematoxylin-eosin (H\&E)-stained images under low (x50) and high (x200) magnification. (B) Safranin O-fast green-stained images under low (x50) and high (x200) magnification. LPS, lipopolysaccharide. The high magnfication images are representative of the insets in the low magnification images.

treatment with LPS led to decreased staining in the ECM area, with only NP cells and skeleton-like ECM left, which indicates the depletion of ECM components and disruption of disc matrix structure (Fig. 5A). In Safranin O-fast green staining images, LPS reduced the Safranin O staining intensity of NP, revealing the dereased content of proteoglycan (Fig. 5B). Treatment with crocin attenuated LPS-induced decreased staining of ECM in $\mathrm{H} \& \mathrm{E}$ staining and Safranin O-fast green staining, maintaining the integrity of the disc matrix structure (Fig. 5A and B). A DMMB assay was used to quantify the proteoglycan content in the NP tissue in the cultured discs. In accordance with the results obtained from histological analysis, the proteoglycan content in the NP tissue was less in the LPS group compared with the control and the LPS + crocin groups (Fig. 6C). Ex vivo experiments on collagen-II, the other important component of the ECM, revealed similar results. By performing immunohistochemical qualitative and quantitative assays, we found that crocin significantly prevented the loss in the collagen-II content in the NP tissue from LPS-induced degradation (Fig. 6A and D). These histological changes demonstrated that crocin significantly inhibited the LPS-induced catabolism of the disc matrix, thereby protecting the disc from degeneration. The results of NBT/DAPI dual staining revealed that cell viability in the discs from all groups, following 7 days of culture, was $>90 \%$ with no significant differences observed among the groups (Fig. 6B and E), which confirms the reliability of the organ culture condition and excludes the ex vivo toxicity of crocin.

\section{Discussion}

Crocin, the main functional component in saffron extracts, has been reported to have various bioactivities in different tissues (42). However, to the best of our knowledge, there are no studies focusing on the effects of crocin on IVD. In the present study, we used LPS to induce IVD inflammation and demonstrated for the first time, to the very best of our knowledge, that crocin effectively prevented the LPS-induced 

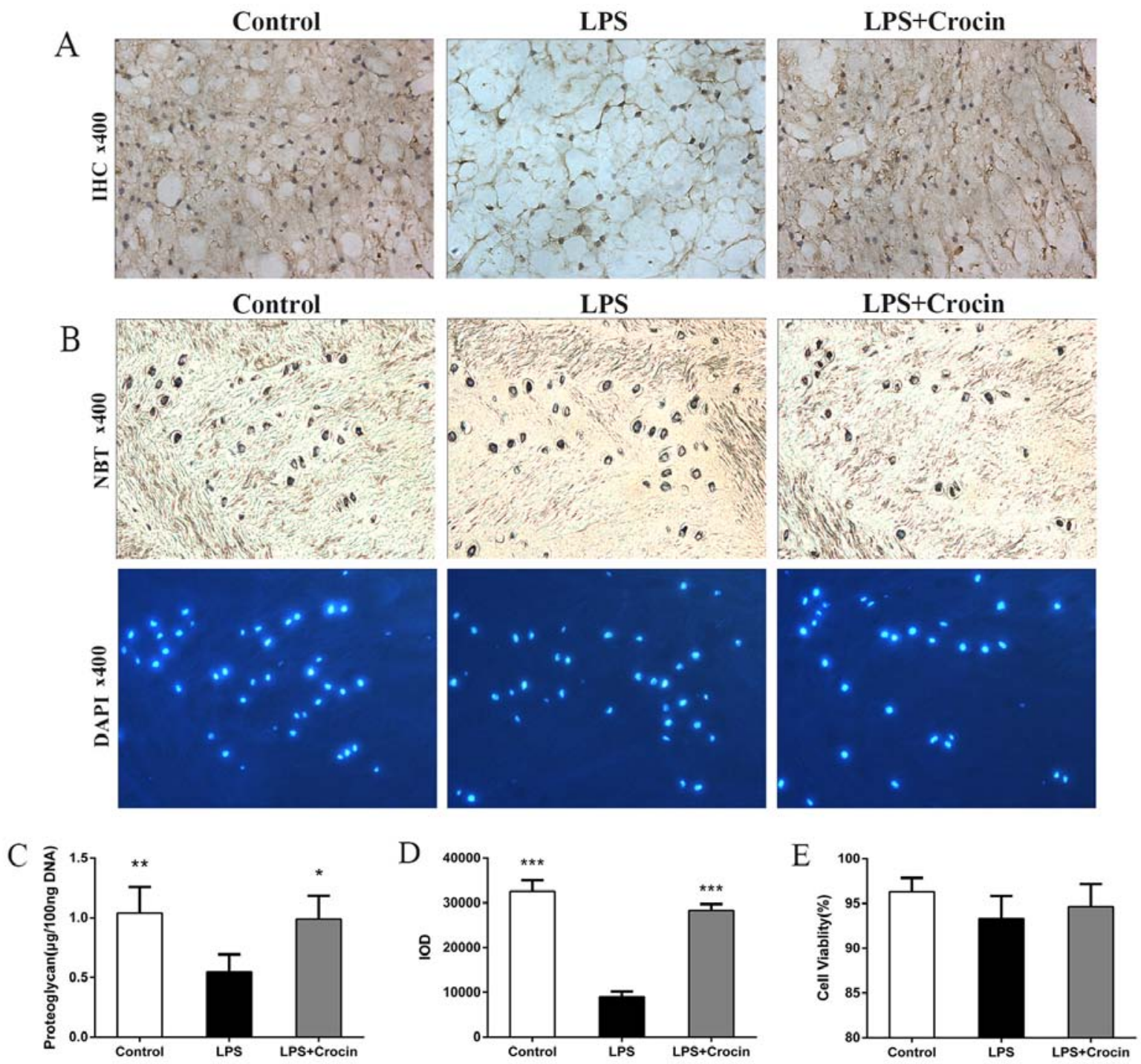

Figure 6. Immunohistochemical, DMMB and cell viability assays of intervertebral discs (IVDs) cultured ex vivo. (A) Collagen-II expression in IVDs (magnification, x400). (B) NBT- and DAPI-stained images (magnification, x400). (C) Proteoglycan content determined by dimethylmethylene blue (DMMB) assay [ ${ }^{*} \mathrm{P}<0.05$ compared to the lipopolysaccharide (LPS)-stimulated group, ${ }^{* * *} \mathrm{P}<0.01$ compared to the LPS-stimulated group]. (D) Quantitative analysis of collagen-II expression using IOD $\left({ }^{* * *} \mathrm{P}<0.001\right.$ compared to the LPS-stimulated group). (E) Cell viability analyzed by NBT/DAPI staining (P $\left.>0.05\right)$.

upregulation of catabolic enzymes and pro-inflammatory factors in the NP in vitro and ex vivo. In addition, the antiinflammatory bioactivity of crocin was shown to be mediated by the suppression of JNK activation.

LPS is a potential inflammatory stimulator and it has been proven to induce the degeneration of the NP (43-47). Evidence indicates that LPS stimulates the expression of catabolic enzymes, e.g., MMP-1, MMP-3, MMP-13, ADAMTS-4 and ADAMTS-5 (44-46). MMPs and ADAMTS degrade aggrecan, the main proteoglycan in the NP, and collagen-II, resulting in the breakdown of the disc matrix (48-51). However, our results demonstrated that crocin markedly suppressed the mRNA expression of MMPs and ADAMTS induced by LPS in a concentration-dependent manner, revealing the anti-catabolic effects of croin on IVDs.

Similarly, several pro-inflammatory factors, such as IL-1 $\beta$, IL-6, TNF- $\alpha$, and oxidative stress factors, such as iNOS, are upregulated by LPS in NP cells (45-47). IL-1 $\beta$ is a well-known pro-inflammatory factor involved in the overexpression of MMPs and ADAMTS, and it contributes to the loss of the disc matrix (52-54). TNF- $\alpha$, another important inflammation mediator, increases the secretion of $\mathrm{NO}$, prostaglandin $\mathrm{E}_{2}\left(\mathrm{PGE}_{2}\right)$ and IL-6 in human NP cells (55). It has been reported that IL-6 not only acts as an independent pro-inflammatory factor, but it also amplifies the effects of IL-1 and TNF- $\alpha$ (56). As regards oxidative stress in the disc, it has been shown that the levels of iNOS, which help to produce NO, highly correlate with IVD degeneration (57). In the present study, we demonstrated that crocin exerts inhibitory effects on the expression of IL-1 $\beta$, IL- 6 , TNF- $\alpha$ and iNOS, which was thought to be associated with the suppressed expression of MMPs and ADAMTS. In accordance with a previous study (47), we found that LPS decreases the expression of aggrecan and collagen-II. As was expected, crocin suppressed the degradation of the ECM components, and partly reversed the downregulation of aggrecan and collagen-II induced by LPS in the NP cells. 
Another noteworthy finding of the present study was that suppressive effects of crocin on TLR-2 expression, antagonizing the effects of LPS. TLRs play a role in early host defense against exogenous pathogens (58) and cartilage inflammatory diseases (59). In particular, TLR-2 has been found to be involved in matrix degradation and in the suppression of matrix synthesis in articular cartilage (60). In the present study, TLR-2 expression was significantly increased by LPS stimulation and inhibited by crocin.

As mentioned above, crocin exerted significant antiinflammatory and anti-catabolic effects in NP cells. Thus, the signaling pathway possibly involved was detected by western blot analysis. The MAPK signaling pathways are actively involved in inflammatory responses (25). Thus, in the present study, the phosphorylation of all 3 MAPKs, including JNK, p38 and ERK, was found to be induced by LPS. However, the activation of JNK was significantly suppressed by crocin, shedding light on a possible mechanism behind its anti-inflammatory effects. Of note, crocin exerted no obvious inhibitory effects on the phosphorylation levels of p38 and ERK, which is possibly due to the multiple roles of p38 and ERK in many other cellular functions, such as cell cycle progression (61), cell growth and differentiation (62).

To further cofirm the effects of crocin, an ex vivo experiment was also performed. LPS was used to induce inflammation and degeneration of the IVDs ex vivo as previously described (46). Previous findings and our results prove that the organ culture condition adopted in the present study allows LPS and crocin, which permeate through the endplate and annulus fibrosus, to exert effects on NP in 7 days of culture (46). Histological and biochemical assessments demonstrated that LPS effectively induced the depletion of the ECM components in the NP tissue, including proteoglycan and collagen-II. However, treatment with crocin significantly attenuated the adverse effects of LPS on ECM in NP tissue, which was consistent with our results obtained in vitro.

All of these results indicated that crocin may be a good choice for the treatment of IVD degeneration in the future, making the prevention of IVD degeneration a possiblity. However, relevant animal experiments to mimic the actual IVD degeneration process and to determine the effects of crocin in vivo are warranted. Importantly, due to the lack of blood supply in the IVD, the oral or intravenous administration of crocin may not provide a satisfactory focal drug concentration which is necessary for the drug effects. Further studies are required to determine appropriate administration routes or ideal drug carriers.

In conclusion, the present study demonstrated that crocin potently inhibited LPS-induced inflammation and catabolism in rat NP cells by suppressing the activation of JNK. Additionally, organ culture experiments proved that crocin protected rat IVDs from ECM depletion. In the future, crocin may prove to be an important candidate for the treatment of IVD degeneration and make the early treatment of IVD degenerative diseases a possibility in clinic practice.

\section{Acknowledgements}

The authors appreciate the technical support from Lei Wang, Xiuqiang Liu, Chuan Jiang and Xiuguo Han. This study was supported by a grant from the National Natural Science Foundation of China (81272038).

\section{References}

1. Hart LG, Deyo RA and Cherkin DC: Physician office visits for low back pain. Frequency, clinical evaluation, and treatment patterns from a U.S. national survey. Spine 20: 11-19, 1995.

2. Katz JN: Lumbar disc disorders and low-back pain: socioeconomic factors and consequences. J Bone Joint Surg Am 88 (Suppl 2): 21-24, 2006.

3. Kuslich SD, Ulstrom CL and Michael CJ: The tissue origin of low back pain and sciatica: a report of pain response to tissue stimulation during operations on the lumbar spine using local anesthesia. Orthop Clin North Am 22: 181-187, 1991.

4. Schwarzer AC, Aprill CN, Derby R, Fortin J, Kine G and Bogduk N: The relative contributions of the disc and zygapophyseal joint in chronic low back pain. Spine 19: 801-806, 1994.

5. Nerlich AG, Schleicher ED and Boos N: 1997 Volvo Award winner in basic science studies. Immunohistologic markers for age-related changes of human lumbar intervertebral discs. Spine 22: 2781-2795, 1997.

6. Boos N, Weissbach S, Rohrbach H, Weiler C, Spratt KF and Nerlich AG: Classification of age-related changes in lumbar intervertebral discs: 2002 Volvo Award in basic science. Spine 27: 2631-2644, 2002.

7. Bibby SR, Jones DA, Lee RB, Yu J and Urban JPG: The pathophysiology of the intervertebral disc. Joint Bone Spine 68: 537-542, 2001.

8. Urban JP and Roberts S: Degeneration of the intervertebral disc. Arthritis Res Ther 5: 120-130, 2003.

9. Roberts S, Evans H, Trivedi J and Menage J: Histology and pathology of the human intervertebral disc. J Bone Joint Surg Am 88 (Suppl 2): 10-14, 2006.

10. Le Maitre CL, Pockert A, Buttle DJ, Freemont AJ and Hoyland JA: Matrix synthesis and degradation in human intervertebral disc degeneration. Biochem Soc Trans 35: 652-655, 2007.

11. Zhao CQ, Wang LM, Jiang LS and Dai LY: The cell biology of intervertebral disc aging and degeneration. Ageing Res Rev 6: 247-261, 2007.

12. Bachmeier BE, Nerlich AG, Weiler C, Paesold G, Jochum M and Boos N: Analysis of tissue distribution of TNF-alpha, TNF-alpha-receptors, and the activating TNF-alpha-converting enzyme suggests activation of the TNF-alpha system in the aging intervertebral disc. Ann NY Acad Sci 1096: 44-54, 2007.

13. Shamji MF, Setton LA, Jarvis W, So S, Chen J, Jing L, Bullock R, Isaacs $\mathrm{RE}$, Brown $\mathrm{C}$ and Richardson WJ: Proinflammatory cytokine expression profile in degenerated and herniated human intervertebral disc tissues. Arthritis Rheum 62: 1974-1982, 2010.

14. Weiler C, Nerlich AG, Bachmeier BE and Boos N: Expression and distribution of tumor necrosis factor alpha in human lumbar intervertebral discs: a study in surgical specimen and autopsy controls. Spine 30: 44-53, discussion 54, 2005.

15. Burke JG, Watson RW, McCormack D, Dowling FE, Walsh MG and Fitzpatrick JM: Intervertebral discs which cause low back pain secrete high levels of proinflammatory mediators. J Bone Joint Surg Br 84: 196-201, 2002.

16. Liu MH, Sun JS, Tsai SW, Sheu SY and Chen MH: Icariin protects murine chondrocytes from lipopolysaccharide-induced inflammatory responses and extracellular matrix degradation. Nutr Res 30: 57-65, 2010.

17. Kim KW, Chung HN, Ha KY, Lee JS and Kim YY: Senescence mechanisms of nucleus pulposus chondrocytes in human intervertebral discs. Spine J 9: 658-666, 2009.

18. Huang QQ and Pope RM: The role of toll-like receptors in rheumatoid arthritis. Curr Rheumatol Rep 11: 357-364, 2009.

19. Abdollahi-Roodsaz S, Joosten LA, Roelofs MF, Radstake TR, Matera G, Popa C, van der Meer JW, Netea MG and van den Berg WB: Inhibition of Toll-like receptor 4 breaks the inflammatory loop in autoimmune destructive arthritis. Arthritis Rheum 56: 2957-2967, 2007.

20. Bobacz K, Sunk IG, Hofstaetter JG, Amoyo L, Toma CD, Akira S, Weichhart T, Saemann M and Smolen JS: Toll-like receptors and chondrocytes: the lipopolysaccharide-induced decrease in cartilage matrix synthesis is dependent on the presence of toll-like receptor 4 and antagonized by bone morphogenetic protein 7 . Arthritis Rheum 56: 1880-1893, 2007. 
21. Iacono A, Gómez R, Sperry J, Conde J, Bianco G, Meli R, Gómez-Reino JJ, Smith AB III and Gualillo O: Effect of oleocanthal and its derivatives on inflammatory response induced by lipopolysaccharide in a murine chondrocyte cell line. Arthritis Rheum 62: 1675-1682, 2010.

22. Pålsson-McDermott EM and O'Neill LA: Signal transduction by the lipopolysaccharide receptor, Toll-like receptor-4. Immunology 113: 153-162, 2004.

23. Jasin HE: Bacterial lipopolysaccharides induce in vitro degradation of cartilage matrix through chondrocyte activation. J Clin Invest 72: 2014-2019, 1983

24. Kittlick PD and Engelmann D: Effect of the microbial constituents, LPS and BCG, on the glycosaminoglycans of chondrocyte cultures. Exp Pathol 42: 145-150, 1991.

25. Berenbaum F: Signaling transduction: target in osteoarthritis. Curr Opin Rheumatol 16: 616-622, 2004.

26. Avruch J: MAP kinase pathways: the first twenty years. Biochim Biophys Acta 1773: 1150-1160, 2007.

27. Chung JW, Zeng Y and Wong TK: Drug therapy for the treatment of chronic nonspecific low back pain: systematic review and meta-analysis. Pain Physician 16: E685-E704, 2013.

28. Michaleff ZA, Kamper SJ, Maher CG, Evans R, Broderick C and Henschke N: Low back pain in children and adolescents: a systematic review and meta-analysis evaluating the effectiveness of conservative interventions. Eur Spine J 23: 2046-2058, 2014.

29. Phillips FM, Slosar PJ, Youssef JA, Andersson G and Papatheofanis F: Lumbar spine fusion for chronic low back pain due to degenerative disc disease: a systematic review. Spine (Phila Pa 1976) 38: E409-E422, 2013.

30. Xu GL, Li G, Ma HP, Zhong H, Liu F and Ao GZ: Preventive effect of crocin in inflamed animals and in LPS-challenged RAW 264.7 cells. J Agric Food Chem 57: 8325-8330, 2009.

31. Nam KN, Park YM, Jung HJ, Lee JY, Min BD, Park SU, Jung WS, Cho KH, Park JH, Kang I, et al: Anti-inflammatory effects of crocin and crocetin in rat brain microglial cells. Eur J Pharmacol 648: 110-116, 2010.

32. Kim JH, Park GY, Bang SY, Park SY, Bae SK and Kim Y: Crocin suppresses LPS-stimulated expression of inducible nitric oxide synthase by upregulation of heme oxygenase-1 via calcium/calmodulin-dependent protein kinase 4. Mediators Inflamm 2014: 728709, 2014

33. Ding Q, Zhong H, Qi Y, Cheng Y, Li W, Yan S and Wang X: Anti-arthritic effects of crocin in interleukin-1 $\beta$-treated articular chondrocytes and cartilage in a rabbit osteoarthritic model. Inflamm Res 62: 17-25, 2013

34. Risbud MV, Izzo MW, Adams CS, Arnold WW, Hillibrand AS, Vresilovic EJ, Vaccaro AR, Albert TJ and Shapiro IM: An organ culture system for the study of the nucleus pulposus: description of the system and evaluation of the cells. Spine 28: 2652-2658, discussion 2658-2659, 2003.

35. Ponnappan RK, Markova DZ, Antonio PJ, Murray HB, Vaccaro AR, Shapiro IM, Anderson DG, Albert TJ and Risbud MV: An organ culture system to model early degenerative changes of the intervertebral disc. Arthritis Res Ther 13: R171, 2011.

36. Lim TH, Ramakrishnan PS, Kurriger GL, Martin JA, Stevens JW, Kim J and Mendoza SA: Rat spinal motion segment in organ culture: a cell viability study. Spine 31: 1291-1297, discussion $1298,2006$.

37. Chiba K, Andersson GB, Masuda K, Momohara S, Williams JM and Thonar EJ: A new culture system to study the metabolism of the intervertebral disc in vitro. Spine 23: 1821-1827; discussion $1828,1998$.

38. Chandrasekhar S, Esterman MA and Hoffman HA Microdetermination of proteoglycans and glycosaminoglycans in the presence of guanidine hydrochloride. Anal Biochem 161: 103-108, 1987.

39. Studer RK, Aboka AM, Gilbertson LG, Georgescu H, Sowa G, Vo N and Kang JD: p38 MAPK inhibition in nucleus pulposus cells: a potential target for treating intervertebral disc degeneration. Spine (Phila Pa 1976) 32: 2827-2833, 2007.

40. Kim JH, Studer RK, Vo NV, Sowa GA and Kang JD: p38 MAPK inhibition selectively mitigates inflammatory mediators and VEGF production in AF cells co-cultured with activated macrophage-like THP-1 cells. Osteoarthritis Cartilage 17: 1662-1669, 2009.

41. Wuertz K, Vo N, Kletsas D and Boos N: Inflammatory and catabolic signalling in intervertebral discs: the roles of NF- $\kappa \mathrm{B}$ and MAP kinases. Eur Cell Mater 23: 103-120, 2012.
42. Alavizadeh $\mathrm{SH}$ and Hosseinzadeh $\mathrm{H}$ : Bioactivity assessment and toxicity of crocin: A comprehensive review. Food Chem Toxicol 64: 65-80, 2014

43. Aota Y, An HS, Imai Y, Thonar EJ, Muehleman C and Masuda K: Comparison of cellular response in bovine intervertebral disc cells and articular chondrocytes: effects of lipopolysaccharide on proteoglycan metabolism. Cell Tissue Res 326: 787-793, 2006.

44. Ellman MB, Kim JS, An HS, Chen D, Kc R, An J, Dittakavi T, van Wijnen AJ, Cs-Szabo G, Li X, et al: Toll-like receptor adaptor signaling molecule MyD88 on intervertebral disk homeostasis: In vitro, ex vivo studies. Gene 505: 283-290, 2012.

45. Iwata M, Ochi H, Asou Y, Haro H, Aikawa T, Harada Y, Nezu Y, Yogo T, Tagawa $\mathrm{M}$ and Hara Y: Variations in gene and protein expression in canine chondrodystrophic nucleus pulposus cells following long-term three-dimensional culture. PLoS One 8: e63120, 2013

46. Kim JS, Ellman MB, Yan D, An HS, Kc R, Li X, Chen D, Xiao G, Cs-Szabo G, Hoskin DW, et al: Lactoferricin mediates antiinflammatory and anti-catabolic effects via inhibition of IL-1 and LPS activity in the intervertebral disc. J Cell Physiol 228: 18841896, 2013.

47. Rajan NE, Bloom O, Maidhof R, Stetson N, Sherry B, Levine M and Chahine NO: Toll-Like Receptor 4 (TLR4) expression and stimulation in a model of intervertebral disc inflammation and degeneration. Spine 38: 1343-1351, 2013.

48. Takaishi H, Kimura T, Dalal S, Okada Y and D'Armiento J: Joint diseases and matrix metalloproteinases: a role for MMP-13. Curr Pharm Biotechnol 9: 47-54, 2008.

49. Fosang AJ, Neame PJ, Last K, Hardingham TE, Murphy G and Hamilton JA: The interglobular domain of cartilage aggrecan is cleaved by PUMP, gelatinases, and cathepsin B. J Biol Chem 267: 19470-19474, 1992.

50. Tortorella MD, Burn TC, Pratta MA, Abbaszade I, Hollis JM, Liu R, Rosenfeld SA, Copeland RA, Decicco CP, Wynn R, et al: Purification and cloning of aggrecanase-1: a member of the ADAMTS family of proteins. Science 284: 1664-1666, 1999.

51. Abbaszade I, Liu RQ, Yang F, Rosenfeld SA, Ross OH, Link JR, Ellis DM, Tortorella MD, Pratta MA, Hollis JM, et al: Cloning and characterization of ADAMTS11, an aggrecanase from the ADAMTS family. J Biol Chem 274: 23443-23450, 1999.

52. Yu ZG, Xu N, Wang WB, Pan SH, Li KS and Liu JK: Interleukin-1 inhibits Sox9 and collagen type II expression via nuclear factorkappaB in the cultured human intervertebral disc cells. Chin Med J (Engl) 122: 2483-2488, 2009.

53. Akyol S, Eraslan BS, Etyemez H, Tanriverdi T and Hanci M: Catabolic cytokine expressions in patients with degenerative disc disease. Turk Neurosurg 20: 492-499, 2010.

54. Lee S, Moon CS, Sul D, Lee J, Bae M, Hong Y, Lee M, Choi S, Derby R, Kim BJ, et al: Comparison of growth factor and cytokine expression in patients with degenerated disc disease and herniated nucleus pulposus. Clin Biochem 42: 1504-1511, 2009.

55. Sinclair SM, Shamji MF, Chen J, Jing L, Richardson WJ, Brown CR, Fitch RD and Setton LA: Attenuation of inflammatory events in human intervertebral disc cells with a tumor necrosis factor antagonist. Spine 36: 1190-1196, 2011.

56. Studer RK, Vo N, Sowa G, Ondeck C and Kang J: Human nucleus pulposus cells react to IL-6: independent actions and amplification of response to IL-1 and TNF- $\alpha$. Spine 36: 593-599, 2011.

57. Furusawa N, Baba H, Miyoshi N, Maezawa Y, Uchida K, Kokubo Y and Fukuda M: Herniation of cervical intervertebral disc: immunohistochemical examination and measurement of nitric oxide production. Spine 26: 1110-1116, 2001.

58. Takeda $\mathrm{K}$ and Akira S: Toll-like receptors in innate immunity. Int Immunol 17: 1-14, 2005.

59. O'Neill LA and Dinarello CA: The IL-1 receptor/toll-like receptor superfamily: Crucial receptors for inflammation and host defense. Immunol Today 21: 206-209, 2000.

60. Joosten LA, Koenders MI, Smeets RL, Heuvelmans-Jacobs M, Helsen MM, Takeda K, Akira S, Lubberts E, van de Loo FA and van den Berg WB: Toll-like receptor 2 pathway drives streptococcal cell wall-induced joint inflammation: critical role of myeloid differentiation factor 88. J Immunol 171: 6145-6153, 2003.

61. Chambard JC, Lefloch R, Pouysségur J and Lenormand P: ERK implication in cell cycle regulation. Biochim Biophys Acta 1773: 1299-1310, 2007

62. Krishna M and Narang H: The complexity of mitogen-activated protein kinases (MAPKs) made simple. Cell Mol Life Sci 65: 3525-3544, 2008. 